\title{
Research on the Chaotic Characteristics and Noise Reduction Prediction of Information System Anomalies in Equipment Manufacturing Enterprises
}

\author{
Peng Niu ${ }^{1}$, Yanming Sun ${ }^{2,3, *}$ and Zhuping Gong ${ }^{1}$ \\ 1 School of Business Administration, South China University of Technology, Guangzhou 510641, China; \\ bmniupeng@mail.scut.edu.cn (P.N.); mezpgong@scut.edu.cn (Z.G.) \\ 2 School of Business Administration, University of Guangzhou, Guangzhou 510006, China \\ 3 Research Center for High Quality Development of Modern Industry, Guangzhou University, \\ Guangzhou 510000, China \\ * Correspondence: sunyanming@gzhu.edu.cn
}

Citation: Niu, P.; Sun, Y.; Gong, Z. Research on the Chaotic Characteristics and Noise Reduction Prediction of Information System Anomalies in Equipment

Manufacturing Enterprises.

Sustainability 2021, 13, 4911

https://doi.org/10.3390/su13094911

Received: 1 March 2021

Accepted: 24 April 2021

Published: 27 April 2021

Publisher's Note: MDPI stays neutral with regard to jurisdictional claims in published maps and institutional affiliations.

Copyright: () 2021 by the authors. Licensee MDPI, Basel, Switzerland. This article is an open access article distributed under the terms and conditions of the Creative Commons Attribution (CC BY) license (https:// creativecommons.org/licenses/by/ $4.0 /)$.

\begin{abstract}
As the process of informatization progresses in an equipment manufacturing enterprise, its information system becomes a dissipative structure due to the nonlinear interaction of many factors. The objectives of this study were to help enterprises adopt intelligent manufacturing, realize sustainable development strategies, and understand the operation rules of information systems. For this purpose, this study analyzed an anomaly index time series of an information system in the process of integration. First, the embedding dimension, time delay, average period, and maximum Lyapunov exponent of the time series were calculated. The anomaly index with chaotic characteristics was denoised by combining phase space reconstruction with singular value decomposition (SVD). Finally, a radial basis function (RBF) neural network and local nonlinear method were used to predict the anomaly index of 79 test data points. The case simulation results verify that the anomaly index is affected by changes in basic data, system development, and online migration. One instance of local noise reduction can reveal hidden problems in the actual operations of enterprises, and multiple iterations can extract the actual information of the signals, avoid failures at isolated points, and show a clear attractor structure. Both the RBF neural network and local nonlinear approach are effective prediction methods with low relative errors, but the performance of the latter is superior.
\end{abstract}

Keywords: manufacturing enterprises; anomaly information systems; chaos characteristic analysis; singular value decomposition local noise reduction; RBF prediction

\section{Introduction}

The integration of industrialization and informatization (IOII) (acronym as shown in Table A1 (Appendix A), the same below) is rapidly advancing in Chinese manufacturing enterprises. In the past 10 years, the Ministry of Industry and Information Technology has evaluated, diagnosed, and benchmarked IOII in China. An evaluation index system has been established, and collaborative environments have been promoted by widely popularizing the IOII management system [1-3]. With the implementation of policy guidance and pilot demonstration, by the end of 2019, 151,597 companies (of which only $6.9 \%$ are large and $32.6 \%$ are equipment enterprises) had participated in the evaluation of IOII. However, only $22.8 \%$ of these enterprises have achieved integration improvement and breakthrough innovation. Most of them (52.4\%) are in the single-system stage, and many $(24.8 \%)$ are still in the initial stage. In addition, the penetration rate of digital R\&D tools has reached $69.7 \%$, and the numerical control rate of key processes is $49.7 \%$, but the readiness rate of intelligent manufacturing is only $7.7 \%$. The development level of IOII in equipment manufacturing enterprises has reached 55.6, which is slightly higher than the national level (54.5) (the above data are from Li Jun [4]). These statistics suggest that the overall integration 
effect of IOII is positive, but the degree of intelligent manufacturing is not optimal. The main reasons that Chinese manufacturing is "big but not strong" are primarily related to IOII problems in the industry, such as the shortage of professionals and capital, the lack of a clear information strategy by enterprise managers, a weak manufacturing foundation, and a low level of information [5]. Large-scale equipment manufacturing enterprises have a large market share because they started early, but the production and operation of some enterprises still have relatively low informatization and low digitalization and largely rely on manual ledgers and telephone or mail communication, which significantly affects their efficiency. Although many enterprises have introduced information systems, they lack a unified basic database, resulting in poor connections among subsystems and causing difficulties in production, warehousing, procurement, and other departments [6].

Previous studies have predominantly focused on three general areas. (1) One key research focus is the stability of the manufacturing information system, such as robustness analysis [7], the construction of index systems [8], analysis of process evolution [9-13], evaluation of dynamic models and chaotic characteristics [14,15], and the study of system maintenance and operation fault handling [16,17]. Liang Peipei et al. [9] report that four main factors support the evolution of information systems in service-oriented manufacturing enterprises: demand-pull, technology-push, resource input, and practice changes. The function, performance upgrade, improvement, and update of local systems in information networks occur gradually, whereas the greatest change in the overall function or performance of the system is rapid. The nonlinear interaction among technology, personnel, and enterprise practices in the system is key to forming orderly and dissipative structures. As suggested by Gong Zhuping [10], the manufacturing information system of an enterprise that has experienced a healthy development period evolves from a low-order to high-order structure, which is followed by a dissipative structure and finally a disordered system. The order parameters that affect the operation of a manufacturing information system include product quality, product cost, the accuracy of market forecasts, market agility, the stability of the supply chain, attention to service, and information security. When order parameters have the same weight of influence on the degree of system order, the state of the system is optimal [18]. Zhang Xiaohua [14] selected the three most representative order parameters (product quality, production efficiency, and production cost) at the decision-making level to establish a three-dimensional dynamic model of system evolution. Through an analysis of its chaotic characteristics, they found that the pursuit of a single goal by enterprises leads to chaos, which is the unpredictable, random-like movement of a deterministic dynamic system, because it is sensitive to initial values. The chaotic characteristics of the manufacturing information system were verified by simulating a time series of production efficiency. Li Heng [16] divided information system faults into four aspects-internal applications, data and related codes, documents, and hardware equipment-and proposed maintenance methods for each of them. The results of Yan Longchuan et al. [19] reveal that more than $80 \%$ of information system faults are unavailable middleware services, insufficient disk storage space or data table space, insufficient server performance, and hardware faults. A BP neural network was used to evaluate the system hardware and software status and predict the system capacity, which enables the rapid recovery and automatic processing of information system faults. Zhu Jianhua et al. [20] revealed the chaotic characteristics of information system faults in small and medium-sized manufacturing enterprises by analyzing a fault time series of smart manufacturing information systems. They report that a BP neural network can accurately predict the fault behavior of reconstructed data, whereas general machine learning methods cannot predict chaotic data. (2) Exploring the factors that affect the integration level of IOII in manufacturing enterprises, Lv Yongwei and Baliwei [21] used systems theory to analyze key factors that affect IOII from three aspects: power, support, and environment. Their results indicate that these three aspects comprise market factors, government promotion, intermediary organizations, IT technological progress, coordinated development requirements of the industrial chain, and an innovation atmosphere; professional talents, financial support, 
standardization construction, public service platform, intermediary service system, government and enterprise support; government policy; level of informatization development; informatization penetration rate; and industry development. Lin Li and Ge Jiping [22] assert that IOII is affected not only by information production equipment, core technologies, and industries but also by collaborative management in the information age, which can be promoted through cultural development, platform building, and the collaboration of personnel, resources, and processes. (3) The role of noise in information transmission has been the subject of several studies. Slight fluctuations in the natural frequency of a neural oscillator, the recording/retrieval error of stored information related to standard time, and fluctuations in neurotransmitter concentration are usually regarded as noise by scholars [23]. As a result of the inevitability of fluctuation, the information transmission process in a natural environment is usually affected by noise, especially in biological systems, such as brain signal processing, and signal fluctuation is a typical characteristic of the noise level [24]. Noise correlation is also widely measured and will potentially be affected by the internal noise, and the peripheral noise may have a substantial impact on cortical representation [25]. When chaotic time-series data are measured, the influence of external environmental factors such as measuring tools often introduce noise to the measured data, which conceals the fundamental characteristics of the chaotic system, and as a result, the reconstructed phase diagram of the signal cannot reveal the complete state [26,27].

Given the above research conclusions, we infer that if equipment manufacturing enterprises aim to achieve sustainable development, then the transformation from traditional manufacturing to intelligent manufacturing cannot occur without the deep integration of information technology and industrial technology. In the process of informatization, people, machines, and the environment interact with each other. This nonlinear interaction will cause anomalies in the information system, or the project may even fail. Therefore, based on chaos theory, this paper presents a time-series correlation analysis of the Enterprise Resource Planning (ERP) anomaly index for equipment manufacturing enterprises. The aim of the approach in this study is to detect problems that exist in enterprise production and operation and information construction, provide warnings to enterprise managers and information technology departments, facilitate the smooth function of the enterprise information system, predict anomalous short-term situations in the system, assess the progress of IOII, and provide a steady stream of power that will enable enterprises to achieve sustainable development. The research conclusion offers practical guidance for managing anomalies in the information systems of Chinese equipment manufacturing enterprises intending to integrate industrialization and informatization.

The rest of this paper is organized as follows. Section 2 of this paper focuses on the methods used, including those applied for chaotic characteristic analysis, noise reduction, and prediction. In Section 3, a case analysis is presented. First, the chaotic characteristics of an anomaly index time series are analyzed, and then, the reconstructed phase trajectory matrix is denoised by local SVD. An RBF neural network and simple nonlinear method are used to predict the anomaly index data after denoising. Sections 4 and 5 are the discussion and conclusion, respectively.

\section{Models and Methods}

The methods used in this study are shown in Figure 1, which is divided into three parts. (1) Chaotic characteristic recognition. First, for a one-dimensional time series, the time delay $\tau$, and embedding dimension $m$ are calculated by using the $C_{-} C$ method, and the average period $T_{\text {mean }}$ is calculated by using the fast Fourier transform (FFT). By substituting the obtained $m, \tau$, and $T_{\text {mean }}$ into the Wolf method, the largest Lyapunov exponent (LLE) of the time-series data can be obtained. The value of LLE is used to determine whether the time series has chaotic characteristics: when LLE $>0$, the time series is chaotic. (2) Chaotic noise reduction. For a chaotic time series, a local noise reduction method that combines phase space reconstruction and SVD can greatly reduce the prediction difficulty. If there are a few isolated points in the reconstructed phase trajectory matrix, one-step local noise reduction 
is sufficient; on the contrary, if the number of isolated points is very large, a step-by-step iterative algorithm should be adopted to reduce the influence of these points. (3) Chaotic one-step prediction. Finally, for denoised data, only one-step prediction is needed, which can be performed by the RBF neural network and simple nonlinear algorithms. The specific methods are introduced in the following subsections.

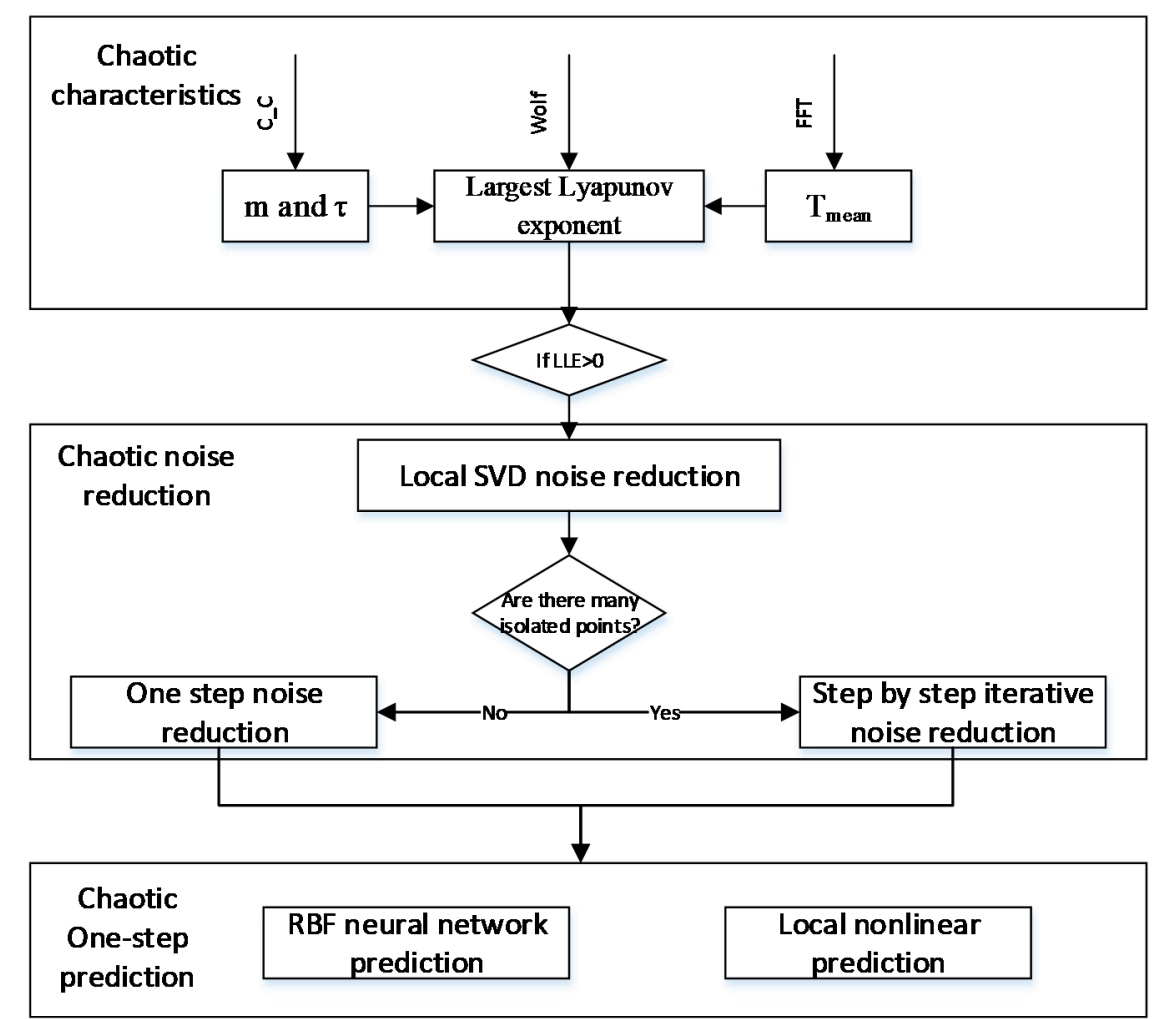

Figure 1. Research methodology framework.

\subsection{Chaotic Characteristic Recognition}

2.1.1. Calculation of the Time Delay and Embedding Dimension by the C_C Method

According to the C_C algorithm proposed by H.S. Kim et al. [28], to reconstruct the time series $x=\left\{x_{i}, i=1,2, \cdots, N\right\}$ (notation as shown in Table A2 (Appendix A), the same below) in phase space, $\mathrm{m}$-dimensional phase space vectors must first be obtained:

$$
X=\left(x_{i}, x_{i}+\tau, \cdots x_{i}+(m-1) \tau\right), i=1,2, \cdots, M, M=N-(m-1) \tau
$$

Then, the correlation integral of the embedded time series should be:

$$
C(m, N, r, t)=\frac{2}{M(M-1)} \sum_{1 \leq i \leq j \leq M} H\left(r-\left\|x_{i}-x_{j}\right\|\right), r>0
$$

where $H(x)=\left\{\begin{array}{l}0, x<0 \\ 1, x \geq 0\end{array}\right.$, which is a Heaviside function. The time series $x$ is divided into $t$ disjoint time series, as shown in Equation (3), where length $l=N / t$, and $t$ is the time scale.

$$
\left\{x_{1}, x_{t+1}, x_{2 t+1}, \cdots, x_{N-t+1}\right\},\left\{x_{2}, x_{t+2}, x_{2 t+2}, \cdots, x_{N-t+2}\right\}, \cdots,\left\{x_{t}, x_{2 t}, x_{3 t}, \cdots, x_{N}\right\}
$$


The test statistics of each subsequence are defined as:

$$
S(m, N, r, t)=\frac{1}{t} \sum_{s=1}^{t}\left[C_{S}(m, N / t, r, t)-C_{s}^{m}(1, N / t, r, t)\right]
$$

When $N \rightarrow \infty$,

$$
\begin{gathered}
S(m, r, t)=\frac{1}{t} \sum_{s=1}^{t}\left[C_{s}(m, r, t)-C_{s}^{m}(1, r, t)\right] \\
\Delta S(m, t)=\max \left\{S\left(m, r_{j}, t\right)\right\}-\min \left\{S\left(m, r_{j}, t\right)\right\}
\end{gathered}
$$

According to the statistical conclusion of BDS, let $m=2,3,4,5, r_{j}=i \sigma / 2, i=$ $1,2,3,4$, where $\sigma$ is the standard deviation of the time series. Then, the estimated quantity is calculated:

$$
\begin{gathered}
\bar{S}(t)=\frac{1}{16} \sum_{m=2}^{5} \sum_{j=1}^{4} S\left(m, r_{j}, t\right) \\
\Delta \bar{S}(t)=\frac{1}{4} \sum_{m=2}^{5} \Delta S(m, t) \\
S_{\text {cor }}(t)=\Delta \bar{S}(t)+|\bar{S}(t)|
\end{gathered}
$$

The first zero point of $\bar{S}(t)$ or the first minimum point of $\Delta \bar{S}(t)$ is the best time delay $\tau$, and the minimum point of $S_{\text {cor }}(t)$ is the best delay time window $\tau_{w}$, so the embedding dimension $m$ can be calculated by Equation (10).

$$
\tau_{w}=(m-1) \tau
$$

2.1.2. Calculation of the Largest Lyapunov Exponent by the Wolf Method

First, the time series is transformed from the time domain to the frequency domain by FFT, and the average period of the original time series can be calculated from the frequency domain information of the transformed sequence. The time series $x(t)=$ $\left\{x\left(t_{i}\right), i=1,2, \cdots, N\right\}$ is subjected to FFT, resulting in:

$$
F(k)=\sum_{n=1}^{N} x(n) e^{-j 2 \pi(k-1) \frac{n-1}{N}}
$$

The frequency used in the transformation is:

$$
f_{n}=2 \pi \frac{n-1}{N}, n=1,2, \cdots, N
$$

The average frequency of the energy spectrum is:

$$
f_{n m}=\frac{\sum_{n=2}^{N} f_{n}}{N}=\frac{2 \pi \sum_{n=2}^{N}(n-1)}{N^{2}}
$$

Therefore, the average period is calculated by the reciprocal of the average frequency:

$$
T_{\text {mean }}=\frac{1}{f_{n m}}=\frac{N^{2}}{2 \pi \sum_{n=2}^{N}(n-1)}=\frac{N}{(N-1) \pi}
$$

Then, the phase space of the time series $x(t)$ is reconstructed to obtain the phase space vector: 


$$
X(t)=\left[X\left(t_{1}\right), X\left(t_{2}\right), \cdots, X\left(t_{M}\right)\right]=\left(x\left(t_{i}\right), x\left(t_{i}+\tau\right), \cdots, x\left(t_{i}+(m-1) \tau\right)\right)^{T}, i=1,2, \cdots, M
$$

With the initial point defined as $X\left(t_{0}\right)$, the short-term separation is limited, and the nearest point $X_{0}\left(t_{0}\right)$ is sought in the range of $\left|X\left(t_{0}\right)-X_{0}\left(t_{0}\right)\right|>T_{\text {mean }}$. The distance between the two points is $l\left(t_{0}\right)$, and the evolution of the two points is tracked from $t_{0}$ until the distance between them exceeds the specified value, which means that $l^{\prime}\left(t_{0}\right)=$ $\left|X\left(t_{1}\right)-X_{0}\left(t_{1}\right)\right|>\varepsilon, \varepsilon$ is the minimum value that is greater than 0 . At this time, $X\left(t_{1}\right)$ is retained; the adjacent points $X_{1}\left(t_{1}\right)$ of $X\left(t_{1}\right)$ are sought, so $l\left(t_{1}\right)=\left|X\left(t_{1}\right)-X_{1}\left(t_{1}\right)\right|<\varepsilon$, and the included angle between $l\left(t_{1}\right)$ and $l^{\prime}\left(t_{1}\right)$ is small. The above process is repeated and iterated backward $H$ times until the end of $X(t)$. Then, the largest Lyapunov exponent is:

$$
\lambda=\frac{1}{t_{h}-t_{0}} \sum_{i=1}^{h} \ln \frac{l^{\prime}\left(t_{i}\right)}{l\left(t_{i-1}\right)}
$$

\subsection{Local Noise Reduction Method Based on Phase Space Reconstruction and SVD}

Since measured data often have noise that conceals the real chaotic dynamic behavior and thus cannot effectively reveal the internal mechanism of system evolution, the error may be large if the prediction is made directly. Thus, it is necessary to first denoise the data to improve the prediction accuracy [29]. The local noise reduction method of phase space reconstruction and SVD is adopted, which can effectively represent the real trajectory and reduce the noise $[27,30]$.

Let the time series of actual data be $s_{t}=x_{t}+\omega_{t}$, where $x_{t}$ is the real signal and the noise signal is $\omega_{t}$. By reconstructing the time series $s=\left\{s_{i}, i=1,2 \cdots, N\right\}$, the phase trajectory matrix is obtained:

$$
D_{m \times(N-(m-1) \tau)}=\left(s_{i}, s_{i}+\tau, \cdots, s_{i}+(m-1) \tau\right), i=1,2, \cdots, N-(m-1) \tau
$$

The steps of one-step noise reduction are as follows:

Step 1: For each phase point, find all adjacent points and their numbers $n_{i}$.

Step 2: When the number of adjacent points satisfies $n_{1} \in\left(\min \left(m, n_{i}\right), a\right), a$ is the given maximum number of adjacent points, and the phase trajectory matrix $C m \times n_{1}$ (composed of the first phase point and its adjacent points in the phase space) is decomposed by:

$$
C m \times n_{1}=\mathrm{U}\left(\begin{array}{cc}
\Sigma & 0 \\
0 & 0
\end{array}\right) \mathrm{V}^{\mathrm{T}}
$$

where $U_{m \times m}$ and $V_{n_{1} \times n_{1}}$ are orthogonal unitary matrices, $\left(\begin{array}{cc}\Sigma & 0 \\ 0 & 0\end{array}\right)_{m \times n_{1}}$ is a diagonal matrix that is unitarily similar to $C, \Sigma=\operatorname{diag}\left\{d_{1}, d_{2}, \cdots, d_{\min \left(m, n_{1}\right)}\right\}, d_{1} \geq d_{2} \geq \cdots \geq$ $d_{\min \left(m, n_{1}\right)} \geq 0$, and $d_{j}$ is the $j$ th singular value of matrix $C$.

Step 3: The first $k\left(0<k<\min \left(m \times n_{1}\right)\right)$ valid singular values of the diagonal matrix $\Sigma$ are selected as the threshold values, and the singular values deviating from the main shape of the signal are set to 0 . Then, the diagonal matrix corresponding to the filtered singular values is subjected to the inverse process of SVD decomposition (18), and after reducing the noise of the phase point, the adjacent point phase trajectory matrix $C^{\prime} m \times n_{1}$ is obtained, which can be updated to $C m \times n_{1}$.

Step 4: Repeat the above process and iterate backward, removing one point at a time until all phase points are completed.

Finally, the time series after local noise reduction is obtained by inverse reconstruction (17), where the time series corresponding to the filtered singular values are regarded as noise signals.

If the neighborhood radius selected by one-step local noise reduction is inappropriate or there are many extreme points in the time-series data, this will result in a large number of isolated points (no adjacent points). This method will not work for these isolated points, 
so it is necessary to re-select or gradually reduce the neighborhood radius for multiple iterations to effectively remove these points. The noise reduction steps of each iteration are consistent with Steps 1-4, as described above.

The phase diagram before and after noise reduction, the signal-to-noise ratio (SNR), and the mean square error (MSE) are obtained to evaluate the noise reduction effect. Generally, the state with the maximum SNR and minimum MSE is indicative of the best noise reduction effect.

$$
\begin{gathered}
S N R=10 * \lg \frac{\sum_{i=1}^{n} x_{i}^{2}}{\sum_{i=1}^{n}\left(s_{i}-x_{i}\right)^{2}} \\
M S E=\frac{\sum_{i=1}^{n}\left(s_{i}-x_{i}\right)^{2}}{n}
\end{gathered}
$$

\subsection{Chaotic One-Step Prediction}

A chaotic time series can be used for short-term prediction but not long-term prediction. As a three-layer feedforward neural network, the RBF neural network has the advantages of global convergence, straightforward determination of the network structure, and quick training process, and it is effective in complex time-series predictions [31,32]. The local nonlinear method can be used to reduce noise $[26,33,34]$, and we tested its prediction ability.

\subsubsection{One-Step Prediction of the RBF Neural Network}

The network topology is shown in Figure 2. First, $k$ groups of delayed reconstructed embedded time-series data $X(1), X(2), \cdots, X(k), m<k<N$ are used to train the neural network. The input nodes of training are:

$$
X(i)=(x(i), x(i+\tau), \cdots, x(i+(m-1) \tau)), i=1,2, \cdots, k
$$

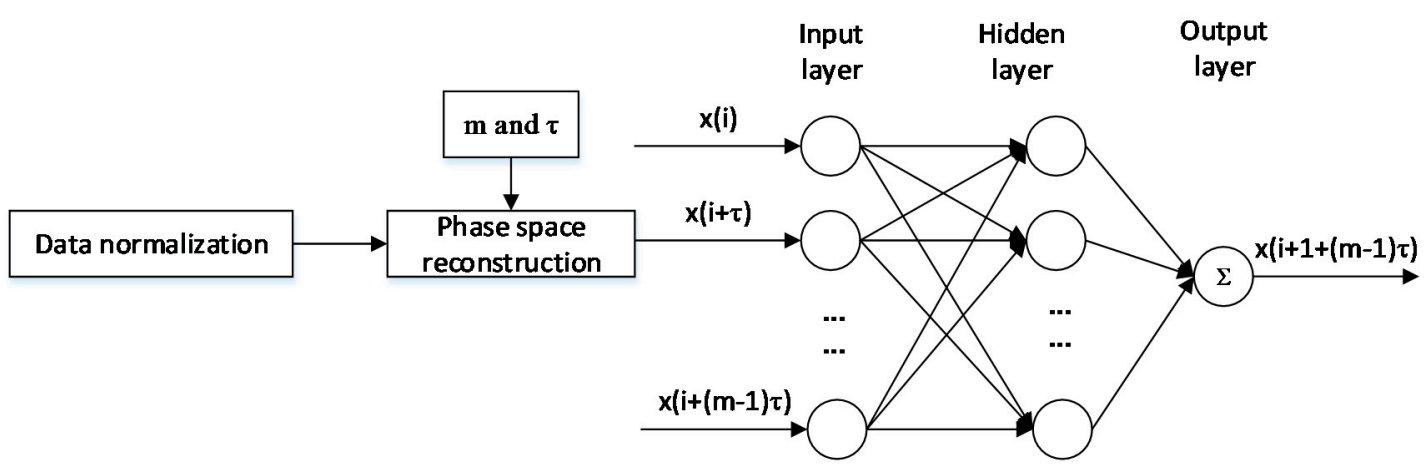

Figure 2. Topological structure of the RBF neural network for phase space reconstruction.

The corresponding ideal output is:

$$
y(i)=x(i+(m-1) \tau+1)
$$

The hidden layer is a nonlinear function $f$, and the output layer is a linear function. If the input of the $i$ th node of the input layer is $x[i]$, then the network model is:

$$
Y(i)=\sum_{i=1}^{m} w(i) f(x[i])=F(X(i))=x(i+(m-1) \tau+1)
$$


The result $\hat{x}$ of one-step prediction should be:

$$
Y(k+1)=\hat{x}(k+(m-1) \tau+2)=F(x(k+1), x(k+1+\tau), \cdots, x(k+1+(m-1) \tau))
$$

Similarly, one-step prediction results $\hat{x}$ of $l(l+k \leq N)$ group test data can be obtained:

$$
\begin{gathered}
Y(k+i)=\hat{x}(k+i+(m-1) \tau+1) \\
=F(x(k+i), x(k+i+\tau), \cdots, \hat{x}(k+i+(m-1) \tau)), i=1, \cdots, l-k
\end{gathered}
$$

The error analysis of the prediction effect is determined by calculating the percentage of relative error $e_{1}$.

$$
e_{1}=\left|\frac{Y(k+i)-y(k+i)}{y(k+i)}\right| \times 100 \%
$$

\subsubsection{Local Nonlinear One-Step Prediction}

For the phase trajectory matrix in Equation (1), all phase points within the neighborhood radius $r$ of each phase point are found. In order to improve the prediction accuracy, the neighborhood radius selected here needs to ensure that none of the phase points are isolated. The average value of the phase trajectory matrix $\mathrm{Cm} \times n_{1}$ (composed of the first phase point $X_{1}$ and its adjacent points) is taken as its predicted value $X_{1}^{\prime}$; the above steps are repeated and iterated backward, removing one point at a time until all phase points are completed. The prediction effect is evaluated by determining the relative error percentage $e_{2}$.

$$
e_{2}=\left|\frac{X^{\prime}{ }_{i}-X_{i}}{X_{i}}\right| \times 100 \%
$$

\section{Case Analysis}

In order to verify the effectiveness of the above methods and clarify the laws and characteristics of information system anomalies in the process of integrating IOII in equipment manufacturing enterprises, the ZZ Machinery Company was studied as an example, and ERP anomalies in the process of informatization development were used in a correlation analysis. In August 2017, the company began to revise part coding, and in June 2019, PDM (Product Data Management) went online. After that, the company implemented automatic coding and rebuilt the 20th BOM (Bill of Material). Therefore, a total of 1248 reports of ERP anomalies were selected from 20160419 to 20200205 . We regard this set of data as representative of ERP anomalies during the informatization development of equipment manufacturing enterprises, including the scenarios caused by changes in basic data, the connection between different systems before and after ERP was brought online, and connection with other modules, such as the supply chain module, finance module, manufacturing module, HR module, salary module, and inventory module. According to the needs of finance, procurement, production, warehousing, and other departments, the ERP and supply chain processes are continually improved so as to analyze problems in the information system while transforming and upgrading from industrialization to informatization. By analyzing these anomalies, we can identify the pace of informatization construction, coordinate the work of different departments to improve the informatization level, and realize the sustainable development of the company. The summary of the anomaly submission form is shown in Table 1.

To facilitate the subsequent mathematical analysis, it is necessary to organize the basic data of ERP anomalies as follows:

Anomaly index $=$ sensitive words ${ }^{*}$ coefficient + anomaly type ${ }^{*}$ coefficient + urgency * coefficient + number of processors ${ }^{*}$ coefficient + processing series ${ }^{*}$ coefficient + processing days $*$ coefficient + processing state * coefficient. 
Table 1. Overview of submission forms of ERP anomalies.

\begin{tabular}{|c|c|c|c|c|c|}
\hline Anomaly Type ${ }^{1}$ & Number & Urgency $^{2}$ & Number & Processor Number ${ }^{3}$ & Number \\
\hline Software fault & 7 & High & 809 & 1 & 299 \\
\hline BOM & 345 & Middle & 162 & 2 & 519 \\
\hline Process & 13 & Low & 277 & 3 & 302 \\
\hline Flow card & 7 & & & 4 & 100 \\
\hline $\begin{array}{l}\text { In and out of } \\
\text { warehouse }\end{array}$ & 126 & & & 5 & 22 \\
\hline Order $^{a}$ & 142 & & & 6 & 4 \\
\hline HR & 7 & & & 7 & 1 \\
\hline Inventory code & 247 & & & 8 & 1 \\
\hline Unknown & 354 & & & & \\
\hline Processing Series 4 & Number & $\begin{array}{c}\text { Processing } \\
\text { Days }{ }^{5}\end{array}$ & Number & Processing State 6 & Number \\
\hline 1 & 456 & $>90$ & 3 & Unable to process & 2 \\
\hline 2 & 574 & 30 90 & 7 & Processing & 88 \\
\hline 3 & 148 & $7 \sim 30$ & 52 & Processed & 1158 \\
\hline 4 & 59 & $3 \sim 7$ & 104 & & \\
\hline 5 & 7 & $1 \sim 3$ & 240 & & \\
\hline 6 & 4 & $<1$ & 842 & & \\
\hline
\end{tabular}

Note: ${ }^{\text {a }}$ Purchase and production orders; ${ }^{1}$ the type of anomaly; ${ }^{2}$ how urgent it was; ${ }^{3}$ how many people it took to resolve it; ${ }^{4}$ how many departments it took to resolve it; ${ }^{5}$ how many days it took to resolve it; ${ }^{6}$ the present status of the anomaly.

Sensitive words include the importance (serious influence, very important, or moderate influence), urgency (very urgent, urgent, as soon as possible, or timely), frequency of occurrence (frequent, often, every time, occasional, or temporary), etc. Each coefficient is set by company executives, ERP administrators, technical center managers, and research group experts. The research data are distributed between 20160419 and 20200205 with different time intervals, and for the convenience of description, the date of the anomaly index is expressed as time $t=\{1,1248\}$. The time series of the ERP anomaly index for informatization construction is shown in Figure 3.

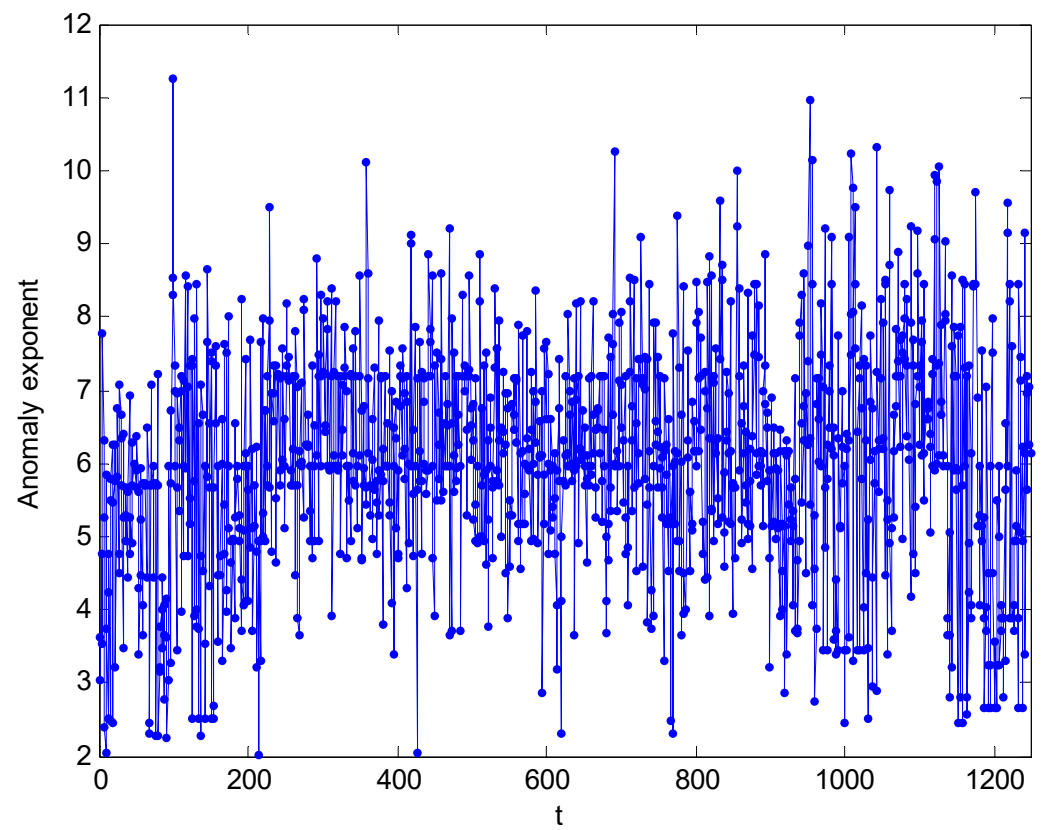

Figure 3. The time series of the anomaly index. 
The time series shows that revising part codes led to a slightly higher anomaly index, especially after PDM went online. This trend is due to frequent conflicts between codes that are automatically generated at that time and the codes from the original system. After a short period of adjustment, the company built a new BOM system to manage the bill of materials as a whole. However, because the internally developed system was poorly connected with other systems, many departments often reported problems related to BOMs.

The chaotic characteristics of this one-dimensional time series were analyzed. First, the time delay and embedding dimension were calculated by the C_C method (see Figure 4), and then, the average period was calculated by FFT (see Figure 5). According to the time delay, embedding dimension, and average period, the Lyapunov exponent of the time series was calculated by the Wolf method (see Figure 6).

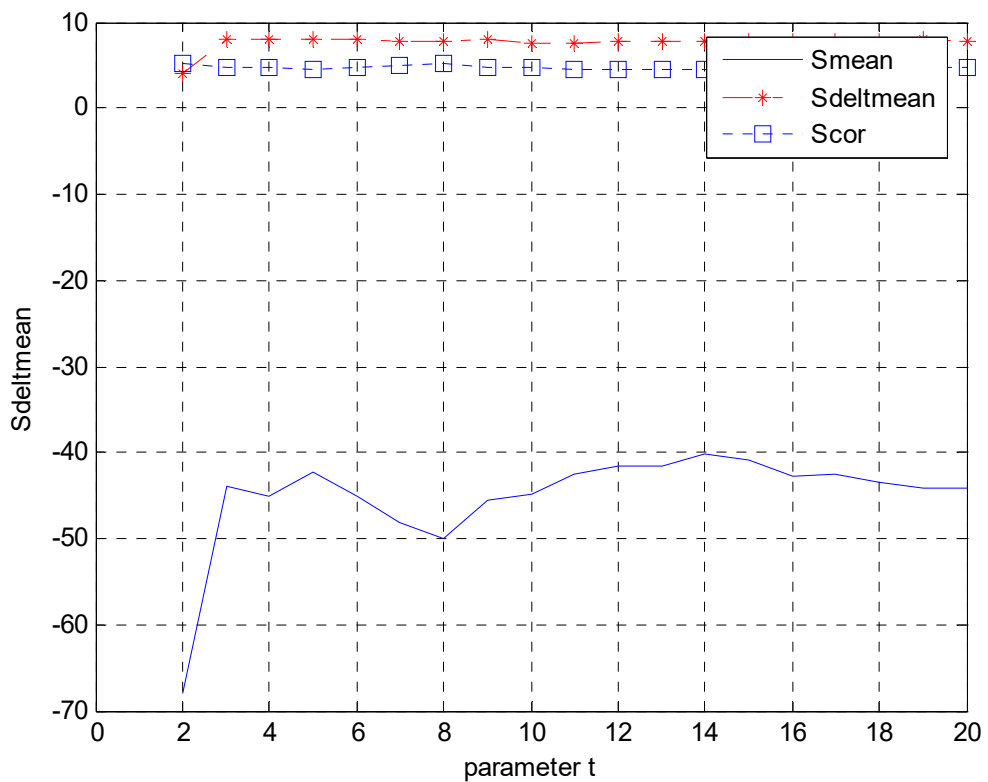

Figure 4. The embedding dimension and time delay determined by the C_C method.
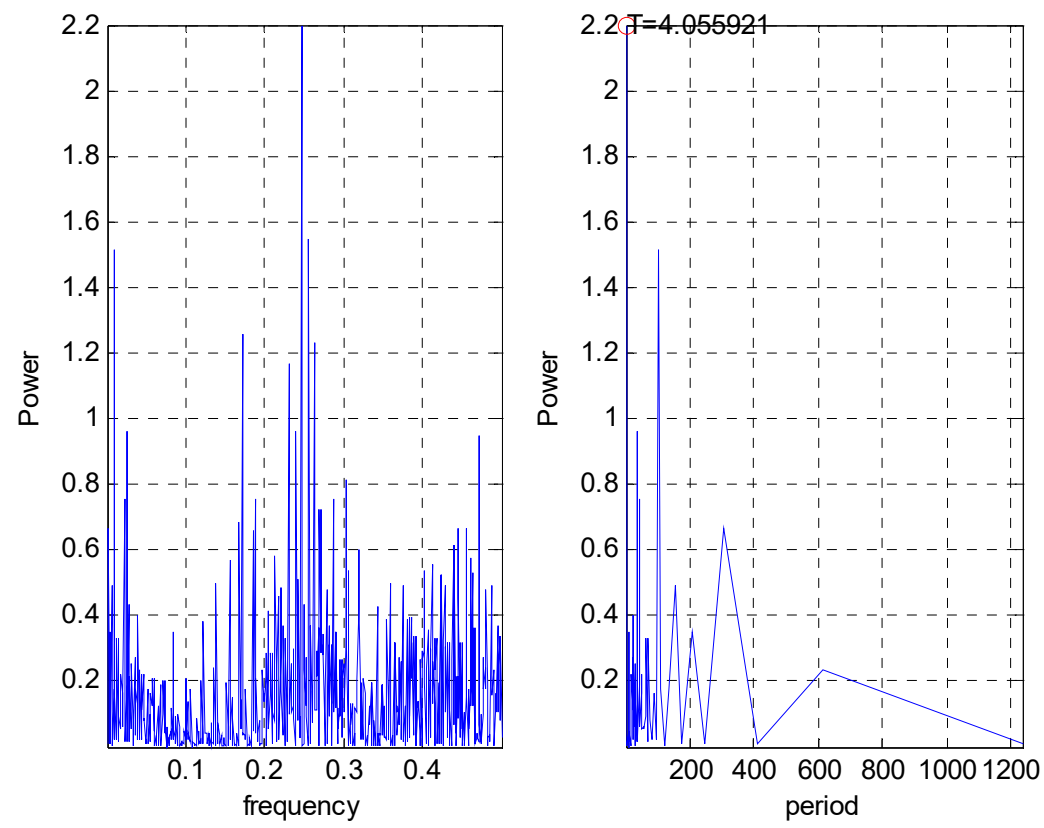

Figure 5. The average period determined by the FFT method. 


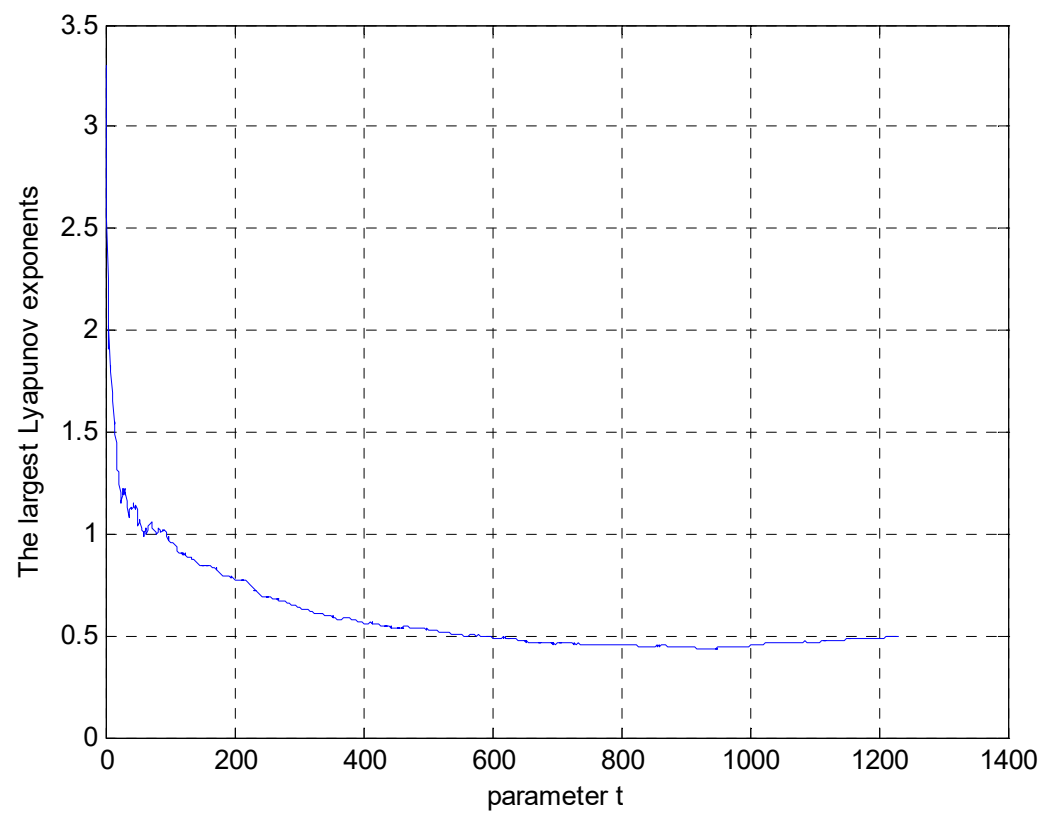

Figure 6. The maximum Lyapunov exponent by the Wolf method.

As revealed in Figure 4, the first minimum value of $\Delta \bar{S}(t)$ appears when $t=5$, and $S_{c o r}(t)$ is the minimum value when $t=14$, so the best time delay is:

$$
\tau=5
$$

and the delay time window is $\tau_{w}=14$. According to Equation (10), the embedding dimension is:

$$
m=1+\left\lceil\tau_{w} / \tau\right\rceil=4
$$

As shown in Figure 5, the average period is 4 . Since the largest Lyapunov exponents are all positive numbers (see Figure 6), and the average largest Lyapunov exponents is 0.5979 , the time series has chaotic characteristics [35], which can be analyzed and predicted by chaos theory.

Since the manual input of anomalies is prone to errors, which will inevitably reduce the prediction accuracy, it is necessary to denoise the time-series data before the prediction. A local denoising method that combines phase space reconstruction and SVD can effectively extract the actual signals of the time series and ensure the reliability of the data.

First, according to the time delay and embedding dimension obtained by the C_C method, the phase space of the time series is reconstructed with $m=4, \tau=5$ (Equations (28) and (29)). The maximum distance of each phase point is $d_{\max }=12.9172$, so the neighborhood radius gradually decreases from 6.5 to 0.1 with a step size of 0.05 , and the variation in the average number of adjacent and isolated points with the radius is shown in Figure 7. In the left figure, the turning point $r=1.2$ on the curve of the average of adjacent points should be the optimal radius of the local noise reduction. At this time, the average of adjacent points is 20, but there are too many isolated points (see Figure 7, right). To carry out the simulation experiment, the number of adjacent points is taken as $n \in(3,26)$, the average value is set as the threshold value, and a singular value less than the average value is set to 0 . The results show that the effect of one instance of local noise reduction is poor (see Figure 8), and it has no effect on 82 phase points. In particular, the extreme points cannot be denoised. Therefore, it is necessary to re-select the neighborhood radius and carry out step-by-step iterative local SVD denoising to remove isolated points. In the right panel of Figure 7, the denoising without isolated points $r=2.6$ gradually decreases to 0.1 with a step size of 0.1 . The simulation was iterated 26 times, and the phase diagram after denoising is shown in Figure 9. The denoising data were reconstructed inversely 
according to Takens' reconstruction theorem, and the time series after denoising is shown in Figure 10.

The effect of step-by-step iterative SVD local noise reduction based on phase space reconstruction is SNR $=16.1582 / \mathrm{db}, \mathrm{MSE}=0.9137$. According to the above analysis, this method results in a better noise reduction effect, smoother data, and a clearer attractor structure. Since the SVD method is a form of principal component analysis (PCA), the selection of a singular value is analogous to extracting a principal component, which effectively filters the noise signal. Reducing the chaotic noise decreases the difficulty of subsequent predictions, and one-step prediction can produce reliable results.
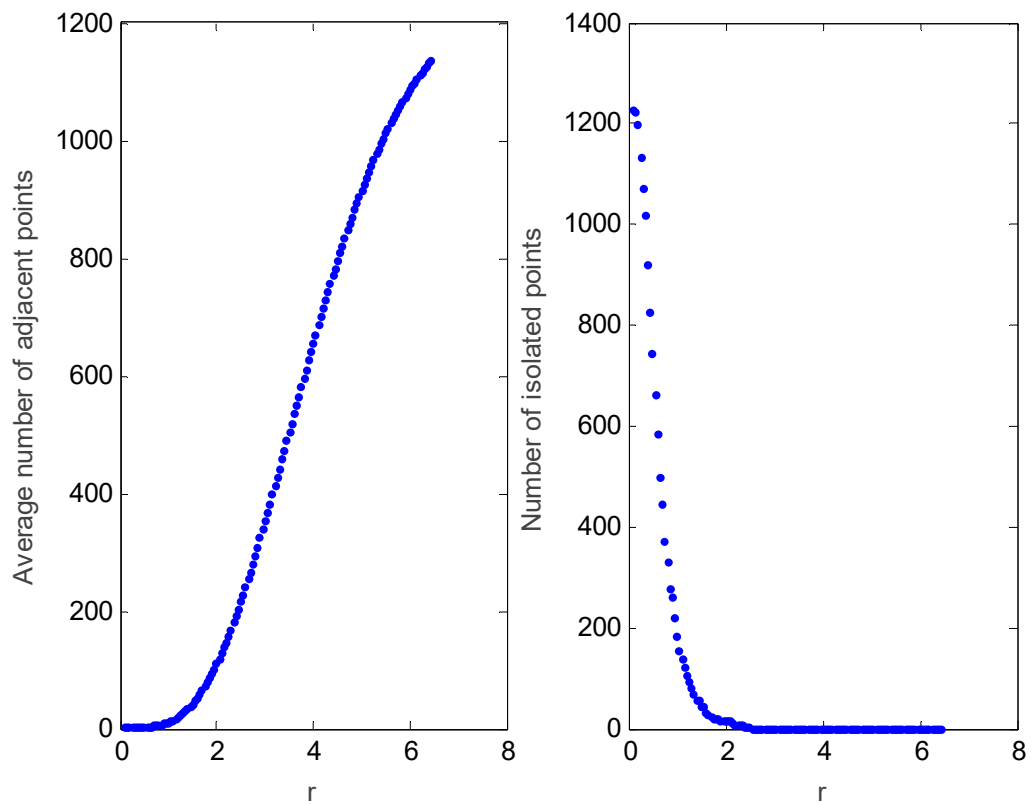

Figure 7. Variation in the average of adjacent and isolated points.

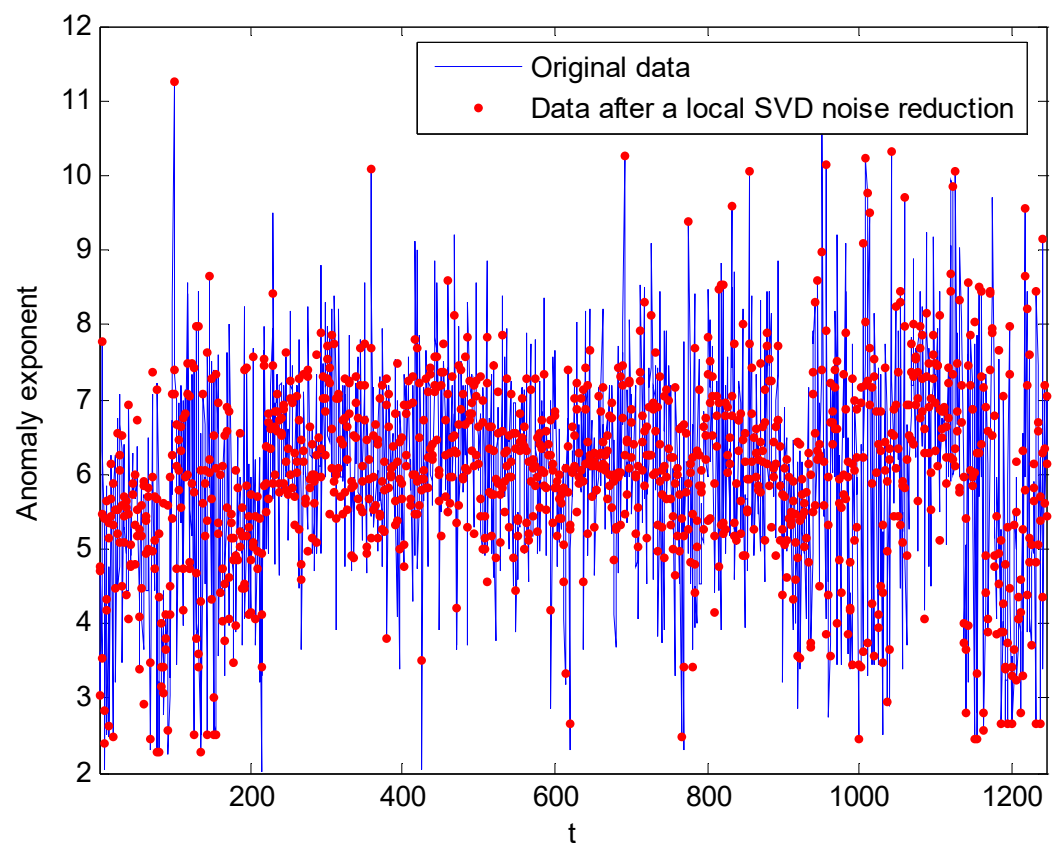

Figure 8. Time-series diagram of one instance of SVD local noise reduction. 

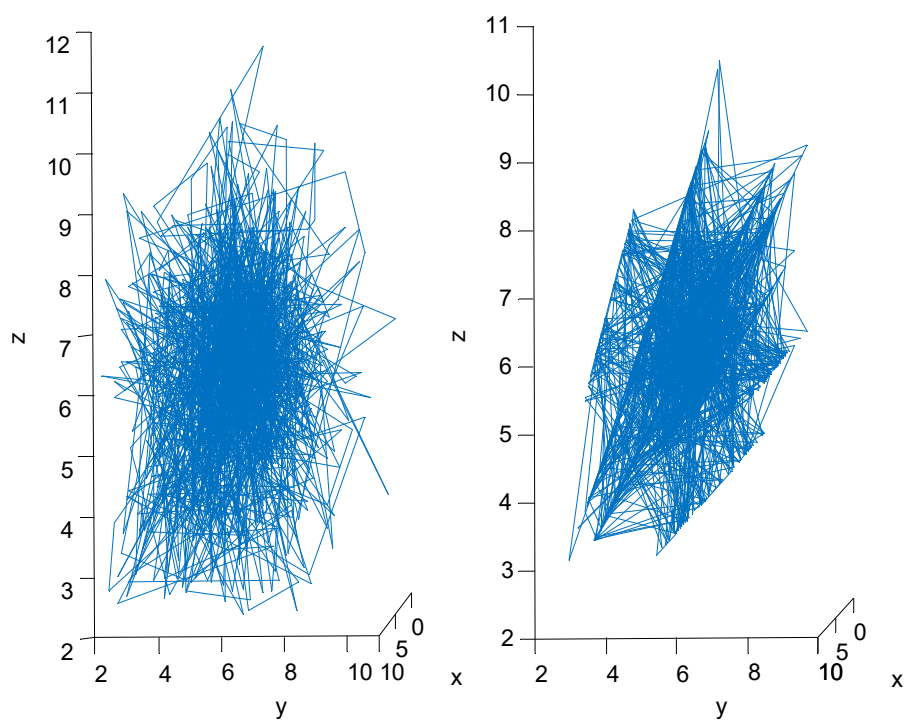

Figure 9. Phase diagram before and after local SVD noise reduction with step-by-step iterative removal of isolated points (the right shows data after noise reduction).

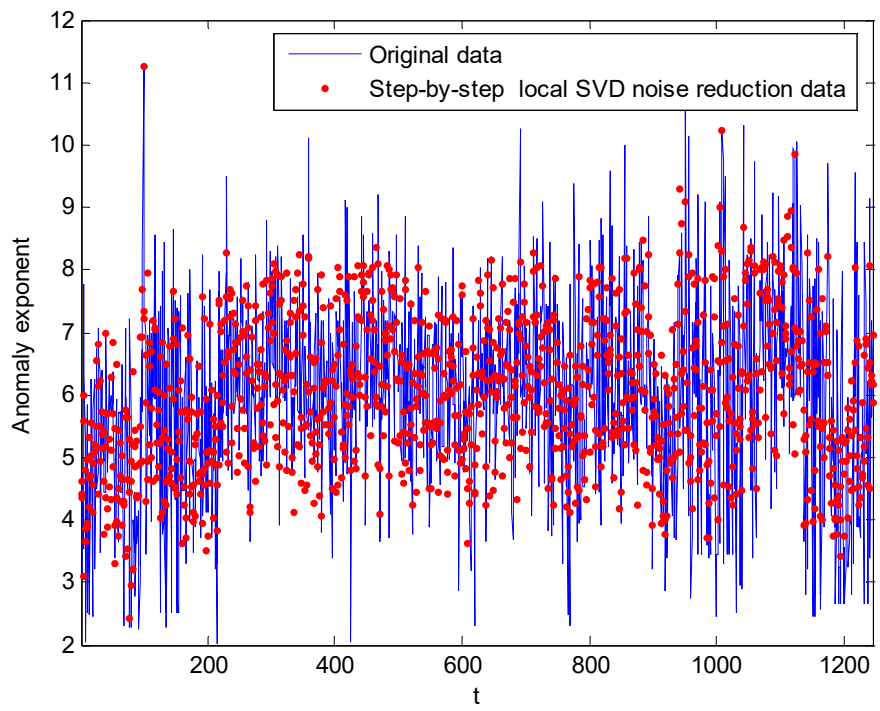

Figure 10. Time-series diagram before and after local SVD noise reduction with step-by-step iterative removal of isolated points.

Then, the RBF neural network is used to predict the local denoising data in one step. The prediction steps are as follows:

Step 1: Normalize the anti-reconstructed data with [0,1].

Step 2: Take the first 500 data points as training samples and the next 79 as test samples, and reconstruct their phase space to obtain two sets of embedded time series and ideal output. Set the dispersion constant of the RBF neural network to 1.

Step 3: Train the RBF neural network using an embedded time series of training samples as input and ideal output as output.

Step 4: Substitute the embedded time series of the test sample into the training network to obtain the one-step-predicted value of the test sample.

The prediction effects of this method were compared with those of the local nonlinear approach. Phase space reconstruction was carried out with the above 79 test samples as research data, and all phase points within the neighborhood radius $r=1.3$ of each phase point were found (the average number of adjacent points in this $r$ was 6, and there were no isolated points). The average value of the phase trajectory matrix (composed of the 
first phase point and its adjacent points) was taken as the predicted value of this phase point, and it was iterated backward, removing one point at a time until all phase points were completed.

The prediction results of the two methods are shown in Table 2, and the relative error curve of the prediction is shown in Figure 11. Table 2 reveals that the one-step-predicted values of the RBF neural network and local nonlinear method are close to the actual values: the absolute errors are between $(-2.09,2.1)$ and $(-0.63,0.65)$, respectively, and the average absolute errors are 0.09 and 0.01 , respectively. Thus, the one-step-predicted results of the local nonlinear method are closer to the actual values, and the predicted values are more stable. The relative errors of the two methods are lower than $0.5 \%$ (see Figure 11). However, the relative errors of the RBF neural network fluctuate considerably, and those of the simple local nonlinear one-step prediction are lower than $0.15 \%$, with small fluctuations and more reliable results.

Table 2. Comparison of phase point prediction results.

\begin{tabular}{llllllllllll}
\hline TRUE & PRBF & \multicolumn{2}{l}{ PNONLTRUE } & PRBF & \multicolumn{2}{l}{ PNONLTRUE } & PRBF & \multicolumn{2}{c}{ PNONLTRUE } & \multicolumn{2}{c}{ PRBF PNONL } \\
\hline 7.16 & 6.30 & 6.88 & 4.50 & 5.23 & 4.76 & 7.22 & 6.60 & 6.74 & 6.71 & 5.74 & 6.59 \\
6.02 & 4.62 & 6.25 & 5.22 & 5.95 & 5.07 & 5.01 & 4.89 & 4.94 & 6.24 & 6.89 & 6.07 \\
6.31 & 7.31 & 6.71 & 6.67 & 6.49 & 6.75 & 4.96 & 5.10 & 4.89 & 7.26 & 7.04 & 7.08 \\
5.42 & 3.82 & 5.48 & 5.55 & 5.71 & 5.66 & 6.00 & 6.54 & 6.13 & 6.46 & 5.38 & 6.53 \\
6.03 & 6.13 & 5.88 & 6.44 & 5.94 & 6.12 & 6.33 & 5.64 & 6.22 & 5.68 & 6.39 & 5.68 \\
6.67 & 5.80 & 6.70 & 6.73 & 5.45 & 6.37 & 5.97 & 5.38 & 6.02 & 4.69 & 5.97 & 4.80 \\
7.64 & 7.01 & 7.00 & 6.68 & 5.65 & 6.59 & 7.16 & 6.02 & 6.90 & 5.20 & 5.57 & 5.33 \\
6.00 & 6.14 & 6.41 & 6.75 & 4.65 & 6.57 & 7.20 & 6.24 & 6.82 & 7.20 & 5.37 & 6.88 \\
5.54 & 4.79 & 6.00 & 5.58 & 6.23 & 5.84 & 5.12 & 5.75 & 5.04 & 6.12 & 5.16 & 6.32 \\
5.56 & 5.66 & 5.55 & 5.45 & 6.27 & 5.67 & 4.75 & 6.38 & 4.78 & 5.68 & 6.63 & 5.49 \\
6.68 & 5.77 & 6.46 & 4.45 & 6.00 & 4.43 & 4.41 & 5.42 & 5.03 & 6.80 & 6.25 & 6.59 \\
6.67 & 5.38 & 6.61 & 7.27 & 5.68 & 6.97 & 6.69 & 6.68 & 6.60 & 5.11 & 4.89 & 5.41 \\
7.11 & 5.38 & 6.51 & 6.59 & 5.50 & 6.20 & 6.48 & 6.51 & 6.26 & 4.91 & 5.35 & 5.00 \\
4.50 & 6.43 & 4.93 & 5.56 & 7.65 & 6.02 & 6.07 & 5.98 & 6.42 & 5.11 & 5.89 & 5.09 \\
5.48 & 6.32 & 5.50 & 5.02 & 6.30 & 5.37 & 6.59 & 5.97 & 6.74 & 6.16 & 5.25 & 6.00 \\
5.21 & 6.07 & 5.21 & 5.90 & 5.80 & 6.11 & 6.03 & 6.05 & 5.84 & 4.97 & 6.24 & 5.15 \\
\hline
\end{tabular}

Note: TRUE is the actual data, PRBF is the predicted data of the test sample with the RBF neural network, and PNONL is the predicted data with the local nonlinear method.

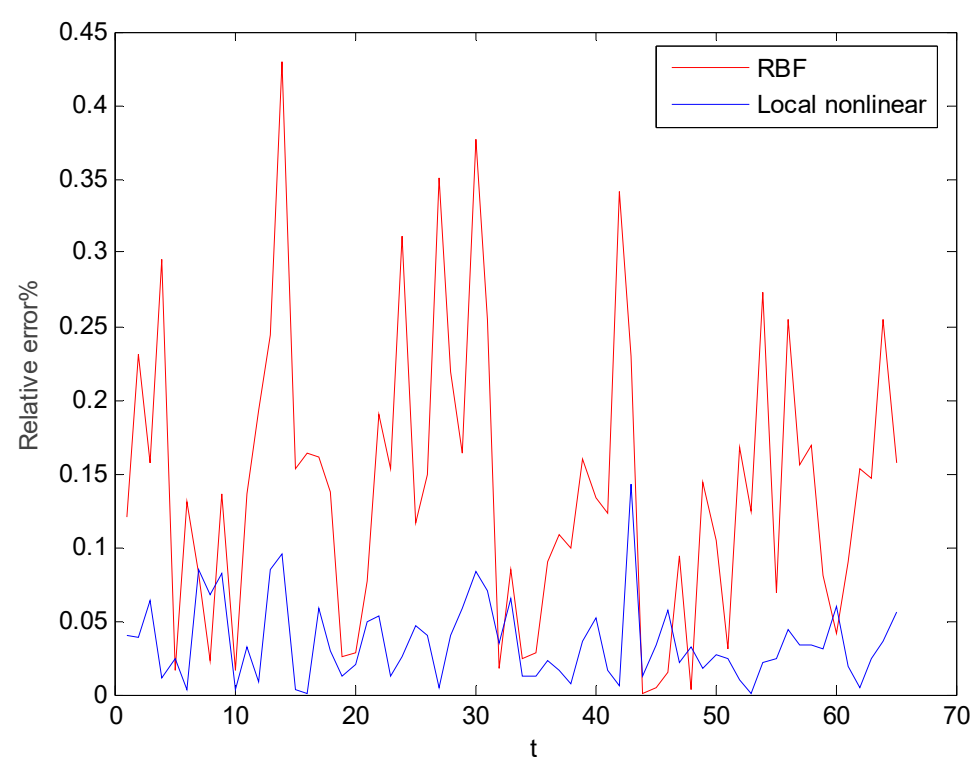

Figure 11. Comparison of relative error curves of phase point prediction methods. 


\section{Discussion}

The results of this paper show that the C_C method can calculate the time delay and embedding dimension of a one-dimensional time series, which provides a basis for subsequent related research. The Wolf method can determine the maximum Lyapunov exponent and its average value of a one-dimensional time series. When the algorithm is implemented, it is necessary to find the average period by FFT, and the maximum Lyapunov exponent is used to determine whether the time series has chaotic characteristics. Reconstruction of the one-dimensional time series in phase space and local singular value decomposition can effectively reduce the noise in the signal and restore its true dynamic trajectory. When there are many isolated points, the noise reduction method for removing isolated points one at a time can be adopted, and the RBF neural network can provide a short-term prediction of reconstructed chaotic data. These results are consistent with the conclusions of previous studies.

The differences are as follows: (1) In the analysis of ERP anomaly data captured during the informatization process in an equipment manufacturing enterprise, we observed that changes in the anomaly index were caused by changes in basic data such as inventory and part coding, the launch of a new system, and the development of a new system. This is because the transformation from industrialization to informatization is a process that involves continuous advancement and upgrading. Traditional manufacturing can realize intelligent manufacturing through a series of operations, such as introducing new equipment, changing business processes and integrating internal and external information systems of the organization. Each step involves many departments and personnel inside and outside the organization. Factors such as the information system itself, developers, the development process and the adaptability of employees once the system is online are bound to cause fluctuations in the anomaly index. (2) When the phase point is denoised, the primary local SVD denoising fails at extreme points (the number of neighboring points, including isolated points, is small), which does not mean that the method is not applicable but rather highlights the advantage of the local SVD denoising method, as it can highlight hidden problems in the enterprise operation at these time points. In fact, due to the lack of uniform standards for inventory names and drawing numbers before the company's PDM went online, the anomaly index was relatively high. In the initial stage of online migration, because the R\&D department forgot to complete the codes in the standard purchased parts list in time, there were many cases in which one item had multiple or missing codes. When the engineer built the BOM, subparts were missing or incorrect, which frequently conflicted with the actual operation. Employees often requested the modification or creation of BOM data for a variety of reasons: for example, the order form and parts list were inconsistent with the actual situation, resulting in an inability to deliver the material from the warehouse; the default warehouse attribute of the inventory file was wrong, and the processed parts that had been transferred to the workshop could not be put into storage; order subparts could not be generated because the BOM was not built to the bottom layer; after the R\&D department issued a design change order, the BOM engineer did not update it in time. Thus, a new BOM system was urgently needed. Subsequently, the company's senior management decided to set up the 20th BOM system for the technical center. The initial problems of the system were mostly caused by inconsistencies in ERP, PDM, U8, and BOM data. It was necessary to strengthen the monitoring of basic data and documents to ensure their accuracy and then try to run MRP on this basis. (3) When forecasting the phase point, the one-step prediction effect of the RBF neural network is good, and the average absolute error is less than 0.1 , but the simple local nonlinear method is superior. Although the relative errors are very small, the latter is smaller and more stable. In terms of computing time, the former takes $1.328 \mathrm{~s}$, and the latter only takes $0.265 \mathrm{~s}$ (the experiment platform was a PC with an Intel Core i5 CPU, 1.8 GHZ and 8 G RAM, and the software was MATLAB_R2015b). Therefore, the simple local nonlinear prediction is superior to the RBF neural network in the prediction of chaotic phase points. Of course, the prediction effect of the neural network is greatly influenced by the training and test 
datasets, especially when there is a large difference between the two, in which case the prediction accuracy will be greatly reduced.

\subsection{Theoretical Contribution}

On the basis of past research, this paper uses chaos theory to discuss problems that arise in an information system during the process of informatization in equipment manufacturing enterprises. The possible contributions are as follows:

(1) The C_C and FFT methods are used to calculate the embedding dimension, time delay, and average period of the ERP anomaly index time series, and the Wolf method is used to calculate its maximum Lyapunov exponent. We found that the ERP anomaly index is generally chaotic due to the nonlinear interaction of many factors. Previous studies have rarely used this research method to study information system anomalies, and research conclusions on this subject are scarce.

(2) When denoising a chaotic time series, phase space reconstruction combined with SVD is effective in local denoising. One instance of local noise reduction fails at an extreme point. Although the noise reduction effect is poor, it can identify important factors that may affect the anomaly index in actual enterprise operations at these time points, which is a unique advantage over other noise reduction methods. Local noise reduction by multiple iterations of isolated point removal prevents failures at extreme points and can reveal a clear strange attractor structure. As SVD is a form of PCA, we infer that this method reveals the real dynamic characteristics of chaotic systems. The feasibility of this method is further verified, and the application range of this method is expanded [27].

(3) Based on the theory of phase space reconstruction, one-step prediction using the RBF neural network and the simple local nonlinear method is applied to the denoised phase trajectory matrix. Both methods have good prediction accuracy, and the local nonlinear prediction effect is more stable. The results further verify the high performance of RBF neural network in chaotic time series prediction [31] and enhance the high prediction efficiency of the local nonlinear method [26].

\subsection{Practical Insights}

This study provides several insights that the management of equipment manufacturing enterprises can use to implement IOII:

(1) Sustainable manufacturing enterprises need a good informatization foundation. The informatization level of independent information systems lacking a unified basic database is clearly not ideal for enterprises, but it is a necessary stage in the transformation and upgrading of most manufacturing enterprises in China. From 2015 to 2019, the development level of IOII in China's equipment manufacturing enterprises increased significantly (12.8\%), but by the end of $2019,74.2 \%$ of equipment manufacturing enterprises were still in the single-system and initial stages [4]. If these enterprises aim to achieve integration and breakthrough innovation, they must inevitably develop (or entrust third-party companies to develop) new information systems that are suitable for their business processes by integrating existing information systems or introducing new integrated information systems. Whether these new systems conflict with the existing systems and whether employees can adapt quickly are the most pressing issues for enterprise managers. Based on the time series of an ERP anomaly index for a case study company, we suggest that equipment manufacturing enterprises with single systems particularly focus on the unity of basic data so as to lay a good foundation for the subsequent development and integration of new systems and related business activities.

(2) An extreme point of a failure in local noise reduction represents the moment at which the most problems arise in enterprise information construction. These problems are largely the result of data inconsistencies, incompatibility between the new and existing systems, and incorrect or omitted operation steps by employees. This suggests 
that sustainable equipment manufacturing enterprises should not only ensure the unification of existing basic data but also coordinate the unification of data between the new and existing systems during the development of new information system projects. This will ensure the timely, accurate, unified, and efficient acquisition, storage and utilization of data between the existing and new systems, and strengthen employee training and guidance in the implementation stage of the new system, thus reducing anomalies and speeding up the development of business processes.

(3) History informs us of successes and failures. Past ERP anomaly indices can predict future ERP operations. Based on phase space reconstruction, the RBF neural network and local nonlinear method can predict the anomaly index after noise reduction. Onestep prediction produces accurate results, which can help enterprise managers predict ERP anomalies in the short term and assess the pace of informatization construction. In addition, managers can adjust the sustainable development strategy of enterprise informatization in a timely manner, and the prediction method can play a role in decision making.

\subsection{Research Limitations}

There are some limitations in this study. In the process of informatization development, the ERP anomaly index could also be influenced by other factors, such as informatization investment, employees' education and technical level, the organizational atmosphere, the industrial synergy, government policy, and enterprise strategy. These issues are not be addressed by this paper. The methods used in this study also have some limitations. For example, the embedding dimension and time delay calculated by the $\mathrm{C}_{-} \mathrm{C}$ method cannot be fully expanded in the reconstructed phase space, and the strange attractor structure is not clear, so other methods should be combined to calculate the chaotic parameters. The prediction effect of the RBF neural network will be affected by training and test data, so the algorithm should be further improved.

\section{Conclusions}

In this study, the embedding dimension and time delay of a time series were calculated using the C_C method, the average period was calculated using the FFT method, and the maximum Lyapunov exponent and its average value were calculated by the Wolf method, which can be used to identify the chaotic characteristics of a time series. Then, the chaotic time series data were denoised by combining phase space reconstruction with SVD, and the denoised data were predicted by the RBF neural network and local nonlinear method. Finally, the chaotic time-series data of the ERP anomaly index captured during the process of informatization development in an equipment manufacturing enterprise were simulated. The results show that the anomaly index is affected by changes in basic data, the development of an information system, and its migration online, which presents chaotic characteristics as a whole. The local SVD method effectively reduces noise. Local noise reduction may fail at extreme points, which can identify jumps in the time series. These jumps may indicate important factors in the enterprise operation that affect the anomaly index, and managers may identify internal problems at these time points. The removal of isolated points and the iterative local SVD noise reduction resolve the failure that occurs with one local SVD instance and can clearly reveal the chaotic attractor structure hidden inside the noise, which can highlight the true dynamic characteristics of the original chaotic system. After noise reduction, one-step prediction can produce reliable results and greatly reduces the difficulty of prediction. The RBF neural network and simple local nonlinear algorithm provide accurate predictions. In particular, the local nonlinear method is worth further research because of its stability and low relative error. In sum, this study used scientific methods to produce results that can serve as a theoretical reference and provide insights for management.

Author Contributions: Conceptualization, P.N.; methodology, P.N. and Z.G.; software, P.N. validation, Y.S., and P.N.; formal analysis, P.N.; investigation, Y.S.; resources, Y.S.; data curation, Z.G.; 
writing—original draft preparation, P.N.; writing—review and editing, P.N.; supervision, Y.S. All authors have read and agreed to the published version of the manuscript.

Funding: This study was supported by the R\&D Project in Key Areas of Guangdong Province [grant numbers 2020B0101050001, Sub-project of man-machine integration, intelligent interaction and knowledge discovery of man-machine interaction and decision-making theory and method in complex manufacturing environment].

Institutional Review Board Statement: Not applicable.

Informed Consent Statement: Not applicable.

Data Availability Statement: The data presented in this study are available on request from the corresponding author.

Acknowledgments: The authors would like to thank the anonymous referees and journal editors for their valuable and constructive feedback.

Conflicts of Interest: The authors declare no conflict of interest.

\section{Appendix A}

Table A1. Acronyms in this paper (ordered by their appearance).

\begin{tabular}{cccc}
\hline Notation & Meaning & Notation & Meaning \\
\hline SVD & Singular value decomposition & MSE & Mean square error \\
RBF & Radial basis function & PDM & Product Data Management \\
IOII & The integration of industrialization and informatization & BOM & Bill of Material \\
ERP & Enterprise Resource Planning & HR & Human resources \\
FFT & The fast Fourier transform & TRUE & The actual data \\
LLE & The largest Lyapunov exponent & PRBF & The predicted data of the test sample \\
& with the RBF neural network \\
SNR & Signal-to-noise ratio & PNONL & The predicted data with the local \\
& & & nonlinear method \\
\hline
\end{tabular}

Table A2. Notation used in this paper (ordered by appearance).

\begin{tabular}{|c|c|c|c|}
\hline Notation & Meaning & Notation & Meaning \\
\hline$x$ & Time series & & The average frequency of the energy spectrum \\
\hline$X$ & Reconstructed phase space vectors of $x$ & $X(t)$ & Reconstructed phase space vectors of $x(t)$ \\
\hline$m$ & Embedding dimension of $x$ & $X\left(t_{0}\right)$ & The point of $X(t)$ at $t_{0}$ \\
\hline$\tau$ & Time delay of $x$ & $X_{0}\left(t_{0}\right)$ & The nearest point of $X\left(t_{0}\right)$ \\
\hline$T_{\text {mean }}$ & Average period of $x$ & $l\left(t_{0}\right)$ & The distance between $X\left(t_{0}\right)$ and $X_{0}\left(t_{0}\right)$ \\
\hline$C(m, N, r, t)$ & Correlation integral of $X$ & $l^{\prime}\left(t_{0}\right)$ & The evolution distance of $l\left(t_{0}\right)$ from $t_{0}$ to $t_{1}$ \\
\hline$H()$. & Heaviside function & $\lambda$ & The largest Lyapunov exponent \\
\hline$S(m, N, r, t)$ & The test statistics of each subsequence & $s_{t}$ & The time series of actual data \\
\hline$C_{s}$ & $\begin{array}{l}\text { The correlation integral of the } s \text {-th } \\
\text { subsequence }\end{array}$ & $x_{t}$ & The real signal \\
\hline$S(m, r, t)$ & Limit value of test statistics when $N \rightarrow \infty$ & $\omega_{t}$ & The noise signal \\
\hline$\Delta S(m, t)$ & $\begin{array}{l}\text { The maximum deviation of } S(m, r, t) \\
\text { when } r \text { takes different values }\end{array}$ & $D_{m \times(N-(m-1) \tau)}$ & The phase trajectory matrix of $s$ \\
\hline $\bar{S}(t)$ & $\begin{array}{l}\text { The average value of } S(m, r, t) \text { with } \\
\text { different } m \text { and } r\end{array}$ & $\mathrm{Cm} \times n_{1}$ & $\begin{array}{l}\text { The phase trajectory matrix composed of the first } \\
\text { phase point and its adjacent points in } D\end{array}$ \\
\hline$\Delta \bar{S}(t)$ & $\begin{array}{c}\text { Average value of } \Delta S(m, t) \text { at } \\
\text { different } m\end{array}$ & $y(i)$ & $\begin{array}{l}\text { The corresponding ideal output of } X(i) \text { in the RBF } \\
\text { neural network }\end{array}$ \\
\hline$S_{c o r}(t)$ & $\begin{array}{c}\text { Statistics representing the optimal time } \\
\text { window }\end{array}$ & $x[i]$ & The input of the $i$-th node of the input layer \\
\hline$\tau_{w}$ & Delay time window of $x$ & $Y(i)$ & The RBF neural network model \\
\hline$x(t)$ & Time series & $Y(k+i)$ & The one-step prediction results of test data for $l$ groups \\
\hline$F(k)$ & Frequency domain of $x(t)$ & $e_{1}$ & $\begin{array}{c}\text { Relative error percentage of the one-step RBF } \\
\text { neural network }\end{array}$ \\
\hline$f_{n}$ & $\begin{array}{l}\text { The frequency used in the FFT } \\
\text { transformation }\end{array}$ & $e_{2}$ & $\begin{array}{l}\text { Relative error percentage the one-step local } \\
\text { nonlinear method }\end{array}$ \\
\hline
\end{tabular}




\section{References}

1. Ma, D.Y.; Jiang, H.Z. Index System Construction for Assessing Integration of Informatizaiton and Industrialization in Enterprise Group. Sci. Technol. Manag. Res. 2019, 39, 212-220.

2. Li, G.; Hu, B. Research on evaluating index and method of enterprise maturity on integration of industrialization and informationization. China Mech. Eng. 2012, 23, 676-680.

3. Li, J.; Qiu, J.J.; Shao, M.K. Current situation, restricting factors and countermeasures study on international competitiveness for China's key technology, products and industrial ecology of the integration of informatization and industrialization. Comput. Integr. Manuf. Syst. 2019, 25, 2334-2343.

4. Li, J.; Qiu, J.J. Research on the Evolution, Progress and Achievements of Integration of Informatization and Industrialization in China between 2014 to 2019. Sci. Technol. Manag. Res. 2020, 40, 175-184.

5. Zhu, J.; Sun, Y.M. Dynamic modeling and chaos control of sustainable integration of informatization and industrialization. Chaos Solitons Fractals 2020, 135, 109745. [CrossRef]

6. Llian, X.Y. Research and Application of Rapid Response Ability Evaluation of Equipment Manufacturing Enterprises; Chongqing University: Chongqing, China, 2018.

7. Song, Z.T. Robustness Analysis and Control of Smart Manufacturing Information Systems; South China University of Technology: Guangzhou, China, 2018.

8. Li, W.C. Construction and Application of Robustness Index System of Service-Oriented Manufacturing Information System; South China University of Technology: Guangzhou, China, 2016.

9. Liang, P.P.; Song, Z.T.; Sun, Y.M. A Research on Complex Information System's Evolution Mechanism of Service-oriented Manufacturing Enterprise. Ind. Eng. J. 2016, 19, 55-62.

10. Gong, Z.P. The Relationship between the Evolvement of Manufacturing Information System and Dissipative Structure. J. Intell. 2007, 1, 79-80+85.

11. Zalozhnev, A.Y.; Zalozhneva, L.L.; Chistov, D.V.; Shuremov, E.L. Evolution and design principles of information systems for enterprise management. Softw. Syst. 2014, 2, 34-38.

12. Fredriksson, O.; Vilgon, M. Evolution of inter-organizational information systems in industrial distribution: The cases of Luna and Pappersgruppen. Eur. J. Inf. Syst. 1996, 5, 47-61. [CrossRef]

13. Hyun, T.; Kim, D.H. The evolution of Korean information infrastructure and its future direction: A system dynamics model. ETRI J. 1998, 20, 1-15. [CrossRef]

14. Zhang, X.H.; Chen, W. Dynamics modeling of manufacturing information system and analysis of chaos. Appl. Res. Comput. 2012, 29, 2978-2981.

15. Gong, Z.P. Comparison of the Calculating Methods of Delay Time in the Reconstructed Phase Space of Manufacturing Quality Information System. Syst. Eng. 2011, 29, 81-85.

16. Li, H. Research on Troubleshooting Based on Information System Maintenance and Operation. Telecom World 2017, 9, 21-22.

17. Liu, J. Discussion on maintenance and operation fault handling of information system. China Comput. Commun. 2016, 20, 47-48.

18. Gong, Z.P. Multiple order parameters evaluation of order-degree in manufacturing information systems with dissipative structure. Comput. Eng. Des. 2009, 30, 4967-4968+5034.

19. Yan, L.C.; Zhang, X.L.; Yang, M. Research and Application of Information System Fault Automatic Recovery Technology Based on BP Neural Network. Electr. Power 2017, 50, 146-149+184.

20. Zhu, J.H.; Gong, Z.P.; Sun, Y.M. Chaotic neural network model for SMISs reliability prediction based on interdependent network SMISs reliability prediction by chaotic neural network. Qual. Reliab. Eng. Int. 2020, 37. [CrossRef]

21. Lv, Y.W.; Ba, L.W. Research on Influence Factors of Integration of Informatization and Industrialization from System Theory Perspective. Chin. J. Syst. Sci. 2014, 22, 84-86.

22. Lin, L.; Ge, J.P. Research of the Two Paths Integration Based on the Concept of Collaborative Management. J. Ind. Technol. Econ. 2012, 31, 30-34.

23. Oprisan, S.A.; Buhusi, C.V. Why noise is useful in functional and neural mechanisms of interval timing? Biomed Cent. 2013, 14 [CrossRef]

24. Pregowska, A. Signal Fluctuations and the Information Transmission Rates in Binary Communication Channels. Entropy 2021 23, 92. [CrossRef]

25. Kanitscheider, I.; Coen-Cagli, R.; Pouget, A. Origin of information-limiting noise correlations. Proc. Natl. Acad. Sci. USA 2015, 112, E6973-E6982. [CrossRef]

26. Gong, Z.P. Local simple nonlinear noise reduction of isolated points in reconstructed phase space. J. Syst. Eng. 2017, 32, 433-440.

27. Niu, P.; Gong, Z.P. Local noise reduction in chaotic time series based on phase space reconstruction and the singular value decomposition. Syst. Eng. 2016, 34, 148-153.

28. Kim, H.S.; Eykholt, R.; Salas, J.D. Nonlinear dynamics, delay times, and embedding windows. Phys. D: Nonlinear Phenom. 1999, 127, 48-60. [CrossRef]

29. Kostelich, S. Noise reduction in chaotic time-series data: A survey of common methods. Phys. Review. Estat. Phys. Plasmasfluidsand Relat. Interdiscip. Top. 1993, 48, 1752-1763. [CrossRef] [PubMed] 
30. Baglio, S.; Fortuna, L. A singular value decomposition approach to detect chaotic dynamics. In Proceedings of the 36th Midwest Symposium on Circuits and Systems, Detroit, MI, USA, 16-18 August 1993.

31. Xia, C.; Wanqin, Y. Prediction and Analysis of Literature Loan Circulation in University Libraries Based on RBF Neural Network Optimized Model. Autom. Control Comput. Sci. 2020, 54, 139-146.

32. Sadiq, A.; Ibrahim, M.S.; Usman, M.; Zubair, M.; Khan, S. Chaotic Time Series Prediction using Spatio-Temporal RBF Neural Networks arXiv. In Proceedings of the 2018 3rd International Conference on Emerging Trends in Engineering, Sciences and Technology (ICEEST), Karachi, Pakistan, 21-22 December 2018; Volume 5, pp. 1-5.

33. Gong, Z.P. Abnormal state in the process of simple nonlinear noise repeated reduction. J. Syst. Eng. 2014, 29, 145-152.

34. Sugihara, G.; May, R.M. Nonlinear forecasting as a way of distinguishing chaos from measurement error in time series. Nature 1990, 344, 734-741. [CrossRef]

35. Liu, S.; Yan, H.; Easa, S.M.; Guo, L.; Tang, Y. Analysis of Stability-To-Chaos in the Dynamic Evolution of Network Traffic Flows under a Dual Updating Mechanism. Sustainability 2018, 10, 4182. [CrossRef] 